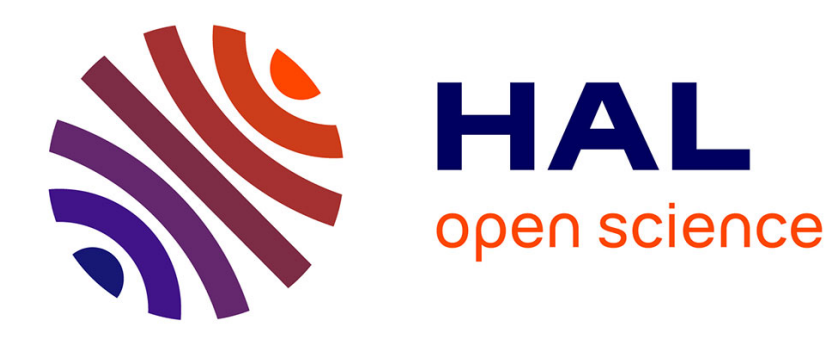

\title{
Vers une écologisation de la gouvernance de la santé
}

Nathalie Blanc

\section{To cite this version:}

Nathalie Blanc. Vers une écologisation de la gouvernance de la santé. Annales des mines - Série

Responsabilité et environnement, 2021, № 104 (4), pp.69-72. 10.3917/re1.104.0069 . hal-03507981

\section{HAL Id: hal-03507981 \\ https://hal.science/hal-03507981}

Submitted on 3 Jan 2022

HAL is a multi-disciplinary open access archive for the deposit and dissemination of scientific research documents, whether they are published or not. The documents may come from teaching and research institutions in France or abroad, or from public or private research centers.
L'archive ouverte pluridisciplinaire HAL, est destinée au dépôt et à la diffusion de documents scientifiques de niveau recherche, publiés ou non, émanant des établissements d'enseignement et de recherche français ou étrangers, des laboratoires publics ou privés. 
Vers une écologisation de la gouvernance de la santé (1)

Nathalie BLANC

Centre national de la recherche scientifique (CNRS), Centre des politiques de la Terre, Laboratoire LADYSS UMR 7533

Les bouleversements dans le champ de la santé sont nombreux, et encore plus lorsque l'on définit la santé comme " un état de complet bien-être, physique, mental et social, qui ne consiste pas seulement en une absence de maladie ou d'infirmité " (voir la charte de l'Organisation mondiale de la santé (OMS)). En effet, la santé ainsi définie l'est plus selon une visée extensive et idéale que par des normes effectivement mises en œuvre. Ces changements relèvent de l'essor mondial des maladies chroniques en relation avec la dégradation de l'environnement (2), de la transformation du rôle de l'hôpital dans la ville, ou encore de la revalorisation du local et du territoire. L'ensemble de ces changements invitent à revoir ce que pourrait être une gouvernance de la santé.

Cette brève réflexion concerne la progressive écologisation des politiques territoriales de santé, en lien avec ces bouleversements. Elle s'inscrit dans une longue histoire, celle des liens entre la santé et l'urbanisme, discipline fondatrice de l'aménagement urbain, ou encore celle de l'intégration de la nature dans l'espace urbain dans l'optique d'une valorisation des qualités des territoires.

De fait, l'écologisation des politiques territoriales de santé n'a cessé d'évoluer au cours des XIXe et XXe siècles.

Par écologisation des territoires, nous fondant ici sur l'introduction du dossier dédié à l'écologisation des territoires coordonné par Ludovic Ginelli, Jacqueline Candau, Sabine Girard, Marie Houdart, Valérie Deldrève et Camille Noûs (3), nous entendons la redéfinition des territoires au travers des normes et des connaissances à visée environnementale. Ces dernières visent à transformer les manières de penser et de juger ainsi que les pratiques sociales sur ces mêmes territoires.

Logiquement, l'écologisation des politiques territoriales de santé impliquerait la prise en compte des normes et des valeurs environnementales par les composantes organisationnelles, culturelles et sociétales du territoire dans la mise en place des politiques de santé. Par local, il ne faut pas entendre forcément un renforcement de la proximité géographique ; l'idée de territoire invite plus largement à repenser les relations, aux différentes échelles géographiques, entre les acteurs, les activités et les milieux de vie. Il s'agit de la mobilisation et de la création d'un grand nombre de ressources, qui peuvent être de différentes natures : économiques, sociales, politiques, culturelles, environnementales, paysagères... Cette écologisation des politiques territoriales de santé implique, à l'aune de la crise environnementale, la redéfinition de la qualité de vie, du bien-être et même de la santé planétaire.

Dès lors, comment ces concepts peuvent-ils être source de nouvelles normes et politiques dans l'occupation des espaces urbains et dans la mise en œuvre de spatialités thérapeutiques ? Dans un premier temps, nous montrerons comment l'histoire de la santé se définit historiquement par la qualité des espaces urbains. Ensuite, nous verrons comment ce contexte se renouvelle à partir de l'émergence de la problématique environnementale dans les débats publics. Il s'agit, notamment, d'une inversion du rôle de la nature et, donc, des modalités d'énonciation des conditions d'un 
habitat valorisant un bien-vivre urbain. Quelle est la place réservée aux mobilisations citoyennes dans cette redéfinition des problématiques de santé au sein des territoires?

\section{Brève histoire}

Au fur et à mesure que se sont développées des interventions publiques en matière de politiques urbaines, notamment à partir du XVIIle siècle, des politiques de contrôle de la nature sont également mises en place (4). Toutes ces interventions s'inscrivent dans un propos d'utilité publique et d'efficacité, " de prophylaxie de l'environnement urbain " (5). On aère, on construit de larges voies, on pave les rues, on permet aux rayons du soleil de pénétrer dans les rues et les maisons, on crée des promenades plantées et des jardins, on déplace les cimetières et les abattoirs hors des villes, on ouvre la ville sur son environnement rural, et on revégétalise les anciennes fortifications. L'air, l'eau et la lumière du soleil circulent et purifient la ville.

Les espaces vacants - les jardins, les bois ou les forêts servant de zones de séparation entre les éléments bâtis - sont une ouverture pour la circulation de l'air et la pénétration des rayons du soleil et, corrélativement, ordonnent l'organisation spatiale de la cité. Mais ils sont aussi des lieux de promenade et de détente. Enfin, la séparation introduite entre les bâtiments par ces mêmes éléments naturels est un moyen pour affecter des fonctions spécifiques à chacun des espaces (usines d'un côté, et habitations de l'autre, par exemple). L'introduction de l'hygiène est également une manière de redonner une force productive aux travailleurs. De l'exploitation de la nature et de son intégration dans le processus de production de la ville, on passe à une sélection des éléments, à un effort pour faire disparaître les inconvénients issus de la nature, grâce au développement des techniques visant à créer une ville qui réponde aux besoins des humains et soit à même d'assurer le progrès social. Ainsi, Benjamin Ward Richardson (1828-1896) (6), médecin anglais, a inventorié dans ce qui fut initialement une communication faite lors du congrès de 1875 de la Social Science Association, les moyens de lutter contre l'état sanitaire déplorable des grandes villes. Dans sa ville fictive d'Hygeia (la Cité de la santé), l'hygiène est la valeur unique qui détermine la localisation et le plan architectural des demeures privées et des édifices publics.

Une véritable maîtrise des effets de la nature dans l'espace urbain, conjuguée à une mise en œuvre technique ébauchant la ville comme un réseau, s'enclenche dans les grandes villes autour des années 1850. En France, ce mouvement va se concrétiser, notamment avec les transformations de Paris sous l'action du Baron Haussmann, alors préfet de la Seine (1853-1870). Les transformations de l'espace urbain vont permettre le développement et la pénétration de techniques urbaines qui favorisent non seulement le confort et l'hygiène, mais aussi l'introduction d'une nature contrôlée.

C'est de la rencontre, vers la fin du siècle dernier et vers le début de celui-ci, de ces deux tendances que sont, d'un côté, l'utopie sociale et, de l'autre, le traitement technique des grandes villes, que I'urbanisme prend corps. Le terme même d'urbanisme entre dans la langue française vers 1900-1910 (7). Le zonage et la pratique du plan vont permettre de réguler les constructions dans l'espace urbain en fonction de principes d'hygiène. II s'agit alors de maîtriser la distance entre les constructions ainsi que l'affectation des espaces urbains. La plupart des urbanistes, proches ou issus du courant moderniste institué par Le Corbusier en 1928 en Suisse et prôné par les CIAM (congrès internationaux d'architecture moderne), cherchent à ordonner les éléments urbains. Critiques de la ville (8), ces penseurs veulent instaurer un espace qui bonifie les conditions d'existence de l'être humain, en mettant en œuvre des principes esthétiques qui tiennent compte de ces nécessités en termes d'hygiène, mais aussi de l'intérêt qu'il y a sur le plan du confort urbain de rationaliser la production du bâti et de la ville. 
De nombreux risques sanitaires pèsent sur les populations en ce XXIe siècle qui ne sont plus les produits d'un environnement miasmatique ou d'une nature pourrissante, mais les résultats d'une morphologie et d'une matérialité biologique, physique et chimique, à l'échelle du bâtiment et de la ville, mais aussi de la région. Un renversement s'élabore : I'hygiénisme, doctrine de l'urbanisme moderne, n'accompagne plus l'idée de progrès. Une ville " propre " n'est même plus une ville saine. Les excès de l'hygiène en zone urbaine s'accompagnent d'une importante pollution atmosphérique, de la prolifération des sols pollués, parfois des décharges à ciel ouvert. Des changements d'optique s'affirment au cours de la décennie 1990 (9). Plusieurs initiatives européennes prennent forme durant la première Conférence européenne sur les villes durables, à Aalborg, en 1994 : le texte de la charte adoptée dans le cadre de cette conférence s'oppose en tous points à celui de la charte d'Athènes (10). La charte d'Aalborg appelle à l'élaboration de politiques urbaines transversales, qui intègrent les impacts du développement sur l'environnement, écologique et social, à plusieurs échelles. Elle met l'accent sur le potentiel des villes et même sur le pouvoir de celles-ci dans la résolution d'un certain nombre de problèmes écologiques et sociaux. Elle les invite à se réapproprier pleinement l'espace politique local, celui de la démocratie urbaine. En 2013, lors de sa présentation du troisième plan national santé-environnement au cours du Conseil des ministres du 12 novembre, la ministre Ségolène Royal a déclaré : « La nature peut être notre premier outil de prévention et notre premier médicament, comme le montrent les bienfaits pour la santé de sa présence en ville et les bénéfices thérapeutiques des jardins en milieu hospitalier (11) ».

La tentation a été grande dernièrement de mettre en œuvre un génie écologique qui se substitue au génie civil et de remplacer les systèmes techniques urbains par des systèmes quasi naturels. Le paysage urbain, du point de vue écologique, doit devenir aussi efficace que l'espace rural. Dès lors, l'urbanisme, né il y a un siècle, commence à se dégager, dans le contexte occidental, bien sûr, de l'emprise hygiéniste et fonctionnaliste lorsqu'il appréhende la question environnementale et sociale ; il reste encore difficile de réfléchir à habiter autrement que ce que prévoyait les cadres de l'urbanisme moderne. La ville a été récemment pensée et bâtie par des professionnels relativement à ses fonctions et à ses usages, en ignorant le fait qu'elle est un milieu de vie et d'épanouissement, en particulier pour les êtres humains non seulement sur le plan fonctionnel, mais aussi dans leur vie de tous les jours, dans le respect de leurs sensibilités différentes. Les citadins ont besoin de faire vivre leurs perceptions et leurs sensibilités, de s'insérer dans un monde allant bien au-delà de la seule sphère productive.

La santé doit s'avérer plus pleinement écologique et sociale. Lorsque les parties prenantes sont diversifiées, les effets combinés de l'écologisation et de la territorialisation semblent plus équilibrés. En ce qui concerne la santé, les acteurs du territoire (associations locales et en réseaux, services publics et territoriaux, populations locales, etc.) et de la qualité des environnements locaux (biodiversité, pollution, etc.) doivent être associés aux acteurs de la santé, pour promouvoir le lien entre les spécialistes thématiques et les acteurs du territoire. Dans ce contexte nouveau où l'environnement est devenu une véritable problématique, les enjeux en termes de santé et de soins ont évolué. Des pratiques voient le jour en contexte hospitalier, lesquelles traduisent plus généralement un rapport au nature modifié. L'écopsychologie, par exemple, désigne un discours sur la psyché en relation avec la Terre, et oblige à se pencher sur la relation entre l'être humain et la nature.

L'exemple de la pollution atmosphérique montre le rôle des associations et des autres acteurs de proximité dans la redéfinition d'une problématique de santé environnementale. Dans les années 1990, de grandes études épidémiologiques sur les liens entre la santé et la pollution ont exprimé les 
risques collectifs (et non pas individuels) avérés de la pollution de l'air ambiant (redécouverte de la pollution de l'air intérieur). La métrologie de la pollution atmosphérique fragmente cette dernière, la caractérise polluant par polluant, et est souvent réalisée loin de la source. Or, les pollutions atmosphériques de proximité liées au tissu urbain dense sont davantage au cœur des nuisances ressenties et dénoncées par les citadins. Les politiques municipales, très attentives à l'époque de l'hygiénisme à l'encadrement des pollutions industrielles, se sont détournées de la question de la pollution atmosphérique, dont elles ont confié la mesure à des réseaux chargés de sa surveillance. Les mouvements sociaux (considérés dans leur dimension collective) accroissent de plus en plus leurs capacités pour être à même de dialoguer avec des scientifiques ou des techniciens. Les plaintes sont territorialisées, renvoient aux sens, et la position de riverain se construit. En somme, la gestion de la pollution perçue est inséparable de la gestion de la pollution nocive qui forme le cœur de la santé environnementale.

\section{Conclusion}

L'aménagement de l'espace et l'urbanisme sont depuis longtemps les outils d'une biopolitique, c'està-dire d'une politique qui régit les rapports des corps vivants entre eux et avec eux-mêmes. Comme l'a dit Michel Foucault, on assiste à partir du XVIIle siècle à " une sorte d'étatisation du biologique ", c'est-à-dire à un investissement par le politique de la vie dans toutes ses dimensions pour la surveiller, la préserver, la réguler... La santé publique devient ainsi l'objet d'une biopolitique définie par l'auteur comme la manière dont on a essayé, depuis le XVIIIe siècle, de rationaliser les problèmes posés à la pratique gouvernementale par des phénomènes propres à un ensemble d'êtres vivants constitués en population : santé, hygiène, natalité, longévité, races... (12) En dépit de sa relation d'opposition avec certains penseurs des utopies alternatives à l'État, les réflexions de Michel Foucault demeurent pertinentes pour comprendre ces utopies urbaines comme étant des postures régulatrices du corps vivant, une régulation mais pas de la même façon qu'un État peut vouloir le faire.

Les origines de cette posture remontent à la seconde moitié du XVIIIe siècle, avec la construction de notions-clés (comme celle de population), l'émergence de nouvelles formes de la connaissance (comme la statistique et la démographie), de nouvelles pratiques (l'hygiène, la sécurité publique, etc.) (13) (Foucault, 2004). Ces différentes notions sont toutes nées en liaison avec l'essor de l'urbanisme moderne conçu en tant que discipline. Agir sur le territoire, c'est agir sur les populations catégorisées comme des corps.

\section{Bibliographie}

(1) Cet article reprend des paragraphes du texte publié en anglais : BLANC Nathalie, "Urban Nature: (The) Good and (The) Bad", in BRETELLE-ESTABLET Florence, GAILLE Marie \& KATOUZIAN-SAFADI Mehrnaz (dirs.), Making Sense of Health, Disease, and the Environment in Cross-Cultural History: The Arabic-Islamic World, China, Europe, and North America, [s.l.], Springer, 2020 (Boston studies in the philosophy and history of science, v. 333), pp. 287-308.

(2) Rapport sur l'état de santé de la population en France en 2017, DREES. 
(3) GINELLI Ludovic, CANDAU Jacqueline, GIRARD Sabine, HOUDART Marie, DELDRÈVE Valérie \& NOÛS Camille (2020), "Écologisation des pratiques et territorialisation des activités : une introduction », Développement durable et territoires [En ligne], vol. 11, n¹, avril, http://journals.openedition.org/developpementdurable/17272, doi: https://doi.org/10.4000/developpementdurable.17272

(4) PERROT Jean-Claude (1974), in RONCAYOLO Marcel \& PAQUOT Thierry (eds.) (1990), Villes et civilisations urbaines - XVIIIe-XXe siècles, Paris, Larousse, coll. " Textes essentiels ", p. 39 : " dans I'optique fonctionnelle, il s'agit de tailler, de greffer, d'amender la nature. Ainsi, une politique populationniste à travers le contrôle des empiriques (sages-femmes, chirurgiens, apothicaires), la surveillance épidémique, la naissance de la médecine sociale (consultations gratuites) ... ».

(5) LUGINBUHL Yves (1992), " Nature, paysage, environnement : obscurs objets du désir de totalité », in Du 731 milieu à l'environnement, Paris, p. 28 : "L'hygiénisme s'instaure en tant que savoir dans le courant du XIXe siècle, savoir technique tout d'abord, savoir scientifique ensuite ».

(6) RICHARDSON Benjamin Ward (1876), Hygeia: A City of Health, London: Macmillan; (2004), Early Urban Planning, vol. 1. Hygeia: A City of Health, ed. Richard LeGates. Reprint Taylor \& Francis.

(7) RONCAYOLO Marcel \& PAQUOT Thierry (eds.) (1990), Villes et civilisations urbaines - XVIIle-XXe siècles, Paris, Larousse, coll. « Textes essentiels ".

(8) L'hygiénisme a inauguré une vision unitaire de la ville comme espace de génération de maladies : le territoire urbain apparaît comme une unité sanitaire : voir MURARD Lionel \& ZYLBERMAN Patrick (1985), " L'ordre et la règle. L'hygiénisme en France dans l'entre-deux-guerres », Les cahiers de la recherche architecturale, $\mathrm{n}^{\circ} 15$ à 17.

(9) EMELIANOFF Cyria (2008), " Enjeux et figures d'un tournant urbanistique en Europe », Annales des Mines - Responsabilité \& Environnement, avril, n52, pp. 15-20.

(10) Élaboré en 1933 lors d'un congrès international d'architecture moderne tenu à Athènes, ce texte constitue le manifeste de l'urbanisme moderne. Il sera publié anonymement en 1942 (Le Corbusier, 1957).

(11) Voir le site de l'Association nationale des villes pour le développement de la santé publique : http://www.espt.asso.fr/activites/participation-au-debat-public/seminaires-colloques-etrencontres/357-5e-congres-national-sante-environnement-organise-par-la-sfse.html

(12) FOUCAULT Michel (2004a), Naissance du biopolitique, Paris, Gallimard/Le Seuil, 688.

(13) FOUCAULT Michel (2004b), Sécurité, territoire, population. Cours au Collège de France (19771978), Paris, Le Seuil-Gallimard, 692. 